\title{
Shaker vibration test of epoxy composites reinforced with pristine and functionalized carbon nanotubes
}

\author{
M. Rafiee*, M. Labrosse \\ Department of Mechanical Engineering, University of \\ Ottawa \\ Ottawa, ON K1N 6N5, Canada \\ mrafiee20@gmail.com,mrafiee@uottawa.ca
}

\author{
F. Nitzsche \\ Department of Mechanical and Aerospace Engineering, \\ Carleton University, 1125 Colonel By Drive, \\ Ottawa, ON K1S 5B6, Canada
}

\begin{abstract}
The vibration and damping characteristics of epoxy composites reinforced with pristine and functionalized multi-walled carbon nanotubes (MWCNTs) were investigated experimentally with potential use as integral passive damping elements in structural composite applications. The MWCNTs were introduced into the acetone solvent and then mixed with epoxy resin through a sonication process and mechanical stirring. The solvent was evaporated by means of magnetic hot plate and the hardener was added to the mixture once it was cooled down to room temperature. The MWCMTs/epoxy mixture was then injected into a mold to form the nanocomposite specimen. Nanocomposite specimens were fabricated for six different MWCNT loadings (0.02, 0.041, $0.061,0.123,0.25$ and $0.37 \mathrm{wt} \%$ ). Microstructural analysis, tensile and bending tests were carried out to examine the effects of pristine multi-walled carbon nanotubes (pMWCNTs) and functionalized multi-walled carbon nanotubes (f-MWCNTs). The frequency response functions (FRFs), coherence and phase diagrams of nanocomposites were measured using a shaker vibration technique. The periodic upchirp signal was generated by a shaker to excite the cantilever nanocomposite specimen at the base. The damped natural frequencies and damping ratios were obtained for different loadings of MWCNTs. The experimental results indicated that the damped natural frequencies of p-MWCNTs/epoxy and fMWCNTs/epoxy composites increased by adding MWCNTs up to 0.12 wt.\% and, after that, decreased with higher MWCNT content. Another finding was that p-MWCNTs were beneficial to improve the damping ratio of nanocomposites. While the damping ratios of f-MWCNTs with loadings of 0.02-0.06 wt.\% were higher than p-MWCNTs ones, they did not increase at higher CNTs contents for the first mode of vibration.
\end{abstract}

Keywords-experimental vibration, carbon nanotubes, polymer composite; vibration damping; functionalize CNTs; epoxy nanocomposite.

\section{INTRODUCTION}

Carbon nanotubes-reinforced polymers have attracted considerable interest due to their potential to exhibit physicochemical properties, such as mechanical, electrical, and thermal properties, that are improved compared to those of their hosting polymers[1-4].

In addition, the investigation of the vibration and damping behavior of composites is an important issue in mechanical and aerospace engineering. Many researchers have studied them both experimentally [5-7] and theoretically [1,7]. A review of state-of-the-art in vibration of composite beams and blades was presented by Rafiee et al. [2]. Studies in the recent years have shown that carbon nanotubes can introduce damping to attenuate the vibrations and noise of two-phase and three-phase epoxy nanocomposites by dissipating substantial amounts of vibration energy [5-7]. However, no study has been reported on the vibration and damping characteristics of functionalized MWCNTs-reinforced epoxy composites.

The present research aimed to understand the practical aspects related to the vibration and damping behavior of pristine and functionalized MWCNTs/epoxy composites with potential use as integral passive damping elements in structural composite applications. In addition, th study also focused on evaluating the effect of MWCNT functionalization on mechanical properties of two-phase epoxy resin composites.

\section{MATERIALS, PROCESSING AND FABRICATION}

\section{A. Materials}

The pristine multi-wall carbon nanotube (NC7000) and amine functionalized multi-wall carbon nanotube (NC7000NH2) were received from Nanocyl S.A., Belgium, with an average diameter and length of $9.5 \mathrm{~nm}$ and $1.5 \mu \mathrm{m}$, respectively. MWCNTs were produced via the Catalytic Chemical Vapor Deposition (CCVD) process with carbon purity of $90 \%$. Low viscosity diglycidyl ether of bisphenol-A (DGEBA) epoxy resin PT-2712 Part A and diethyltoluenediamine (DETDA) curing agent PT-2712 Part B1 produced by PTM\&W Industries, Inc., USA were used. Epoxy 
resin Part A and B mixing weight ratio was 100/22 as per manufacturer recommendation.

\section{B. Processing and fabrication of MWCNTs/polymer nanocomposites}

The two-phase polymer matrix composites reinforced by pristine multi-wall carbon nanotube (p-MWCNTs) or functionalized multi-wall carbon nanotube (f-MWCNTs) were prepared with nanotube loadings of $0.025,0.050,0.075,0.15$, 0.3 and 0.45 of DGEBA wt $\%$. The equivalent nanotube loadings with respect to total weight (DGEBA+DETDA) were $0.020,0.041,0.061,0.123,0.246$ and $0.369 \mathrm{wt} \%$, respectively. Figure 1 shows a schematic of the method for the preparation of the MWCNTs/epoxy nanocomposites.
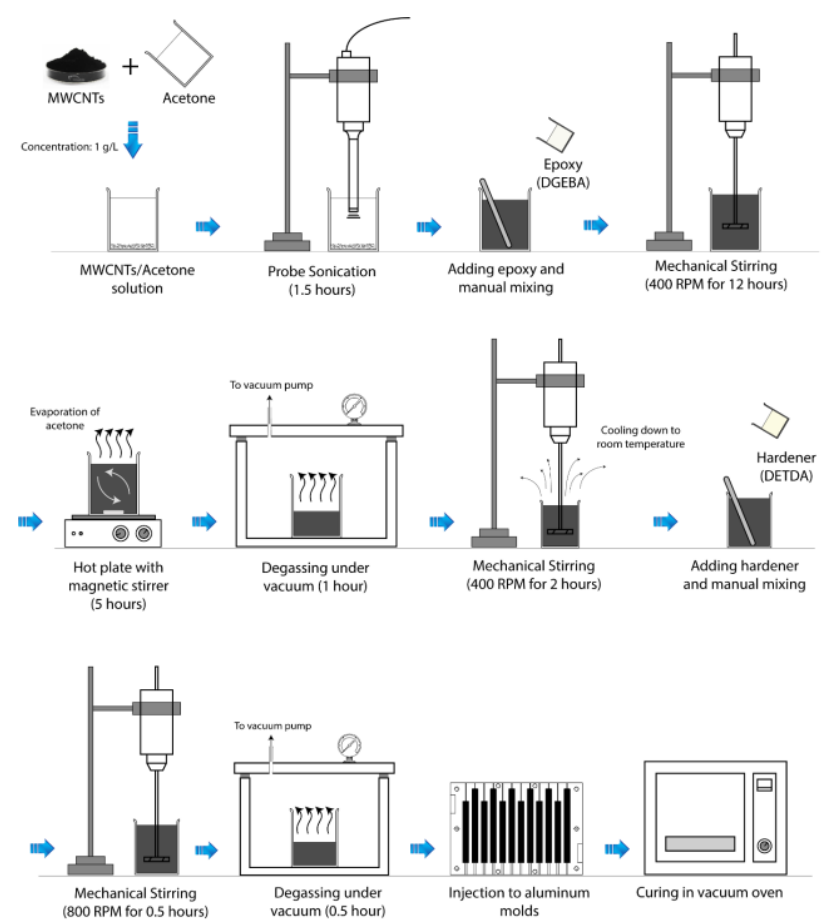

Figure 1. Fabrication process of MWCNTs/epoxy two-phase composites.

The samples were cured at room temperature for 15 hours followed by a post cure of $1^{\circ} \mathrm{C} / \mathrm{min}$ ramp rate, held every $20^{\circ} \mathrm{C}$ for an hour, then continued until a final hold of three hours at $100^{\circ} \mathrm{C}$ to remove the possible thermal history.

\section{EXPERIMENTAL SETUP}

The shaker vibration test was used to measure the natural frequencies and damping factors of nanocomposites specimens in the form of cantilever beams. The clamped-free beam was excited by a vibration shaker (Brüel \& Kjær Sound \& Vibration Measurement A/S, Denmark) at the clamped end, and the response of the beam was tracked by means of accelerometers. The computed frequency response functions (FRFs) gave information about the natural frequencies of the composite beam.

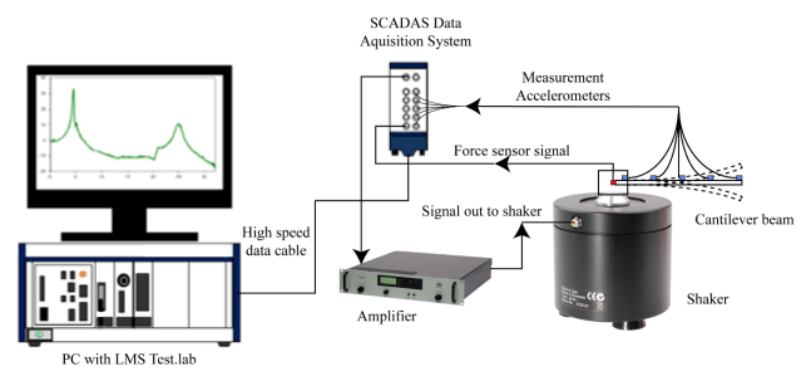

Fig. 2. Schematic diagram of the experimental procedure for vibration testing of MWCNTs- reinforced composites.

Rectangular specimens $12.7 \mathrm{~mm}$ (0.5 in) wide, $3.175 \mathrm{~mm}$ thick (1/8 in) and $152.4 \mathrm{~mm}$ long (6 in) were prepared for vibration tests according to ASTM E756. The free beam length is defined as the distance between the clamp and the specimen tip and was measured as $130 \mathrm{~mm}$. Figure 2 illustrates the instrumentation used for the vibration test. Acceleration was measured at five locations by means of miniature lightweight accelerometers. A piezoelectric crystal force transducer (Quartz ICP® Force Ring, PCB ${ }^{\circledR}$ Model 201B03, measurement range of $2.224 \mathrm{kN}$ ) was used to measure the excitation force exerted by the electrodynamic shaker. A power amplifier was required to drive the shaker. Periodic chirp excitation signals were used with a frequency bandwidth of $0-1024 \mathrm{~Hz}$ to guarantee the highest quality measurements. The tests were performed using the LMS Test.Lab by Siemens ${ }^{\circledR}$, which is an integrated solution for experimental vibrational analysis including tools for data acquisition (LMS SCADAS III with eight measurement channels and two output channels), test execution and data processing (LMS Testing Solution Software). The LMS SCADAS III was used to acquire the signals from the force sensor and the piezoelectric accelerometers and to control the amplifier connected to the shaker, by means of a digital-analog converter output. Spectral Testing module of LMS Testing Solution Software was used for shaker test. The Modal Analysis module was then used to analyze the power spectral density (PSD) of the excitation signal, the FRFs and the coherence functions, and to calculate FRF sum functions (i.e. the sum of the five FRFs). Finally, the PolyMAX frequency domain identification method [27], which is based on a least squares fit, was used to identify the natural frequencies, damping ratios and vibration modes of MWCNTs/epoxy nanocomposites. All measurements were acquired in the frequency range $10-1024 \mathrm{~Hz}$, with a resolution of $0.125 \mathrm{~Hz}$. Each measurement was averaged over 20 acquisitions.

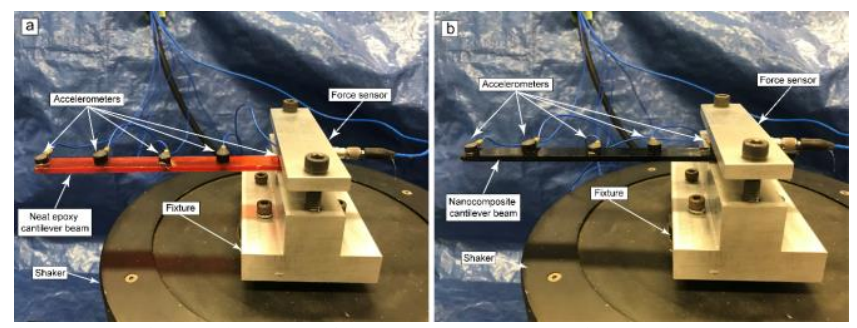

Fig. 3. Experimental set-up with (a) neat epoxy specimen, (b) nanocomposite specimen. 


\section{RESULTS AND DISCUSSION}

Damped natural frequencies, frequency-response functions (FRFs), phase diagram, coherence, and damping ratios about the first three vibration modes were obtained and shown in Figures 4 through 7.
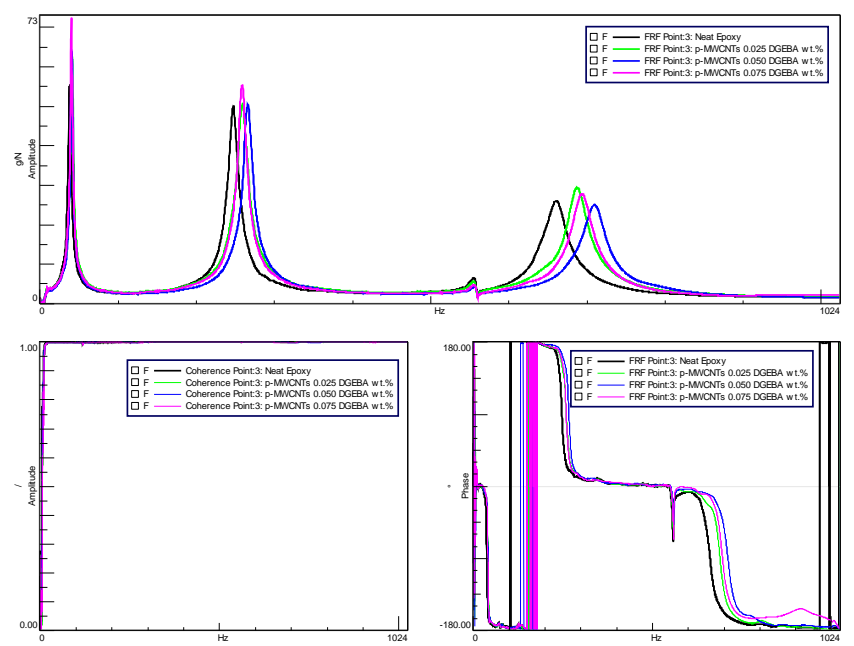

Fig. 4. FRFs, coherence and phase diagrams for neat epoxy and p-MWCNTs $(0.025,0.050$ and 0.075 DGEBA wt.\%) reinforced composites

As can be seen, the damped natural frequencies of pMWCNTs/epoxy and f-MWCNTs/epoxy composites increased by adding CNTs up to $0.12 \mathrm{wt} . \%$, and decreased after that with higher MWCNTs content. The maximum damping improvement of $9.4 \%$ happened at $0.02 \mathrm{wt} \%$ of $\mathrm{f}$ MWCNTs/epoxy composites while it was $7.2 \%$ with the equivalent pristine MWCNTs. The improvement in damping of the fundamental natural frequency was more significant than that at higher natural frequencies. For instance, addition of $0.02 \%$ of f-MWCNTs improved the damping of the fundamental, second and third natural frequencies by $9.4 \%$, $7.5 \%$ and $6.3 \%$, respectively.
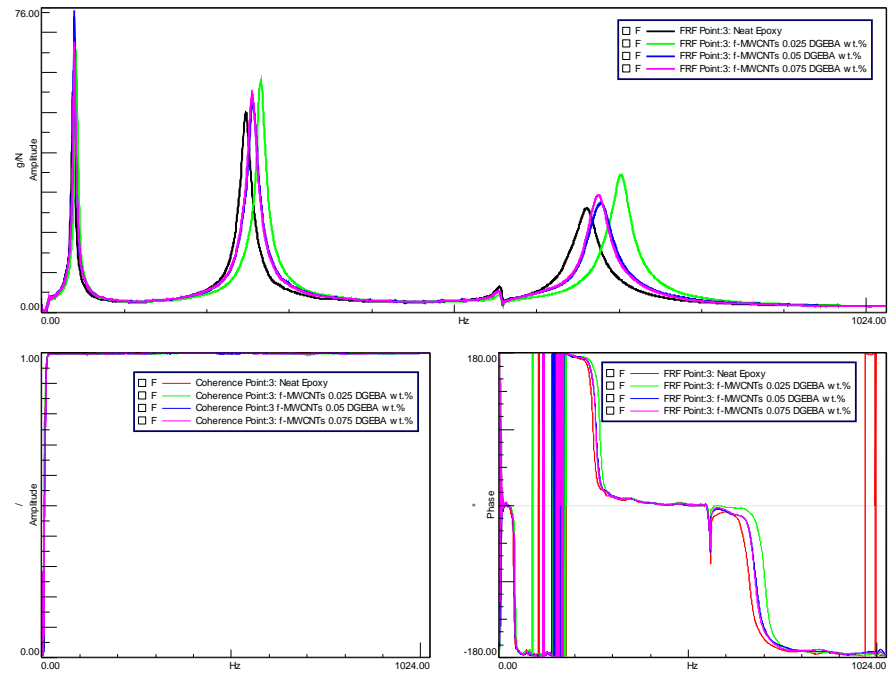

Fig. 5. FRFs, coherence and phase diagrams for neat epoxy and f-MWCNTs $(0.025,0.050$ and 0.075 DGEBA wt.\%) reinforced composites
The relative effects of pristine vs. functionalized carbon nanotubes on the damping ratios of epoxy-based composite beams were investigated and are depicted in Fig. 6 and 7, respectively. As one can observe from Fig. 6, the damping ratios dropped by $18 \%$ till loading of 0.06 wt. $\%$ and then improved by adding $\mathrm{p}-\mathrm{MWCNTs}$ in the composites for the first vibration mode. A $46 \%$ improvement in damping ratio of $p$ MWCNTs/epoxy composite was observed with a loading of 0.37 wt.\%. For f-MWCNTs/epoxy composites, a decrease in damping ratio can be observed over the whole range of loadings While the damping ratios with f-MWCNTs at loadings of 0.02-0.06 wt.\% were still higher than those with pMWCNTs, they did not increase with higher CNT contents for the first mode of vibration.

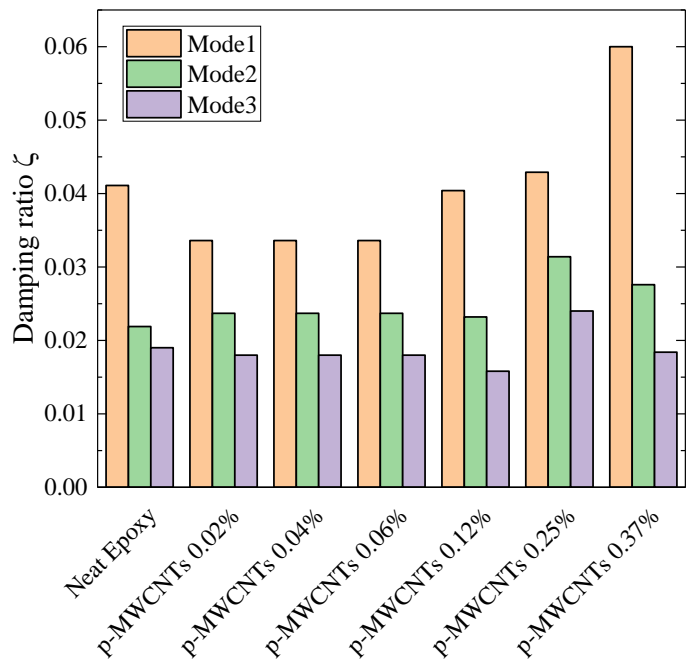

Fig. 6. Damping ratio of p-MWCNTs-reinforced composites with different loadings.

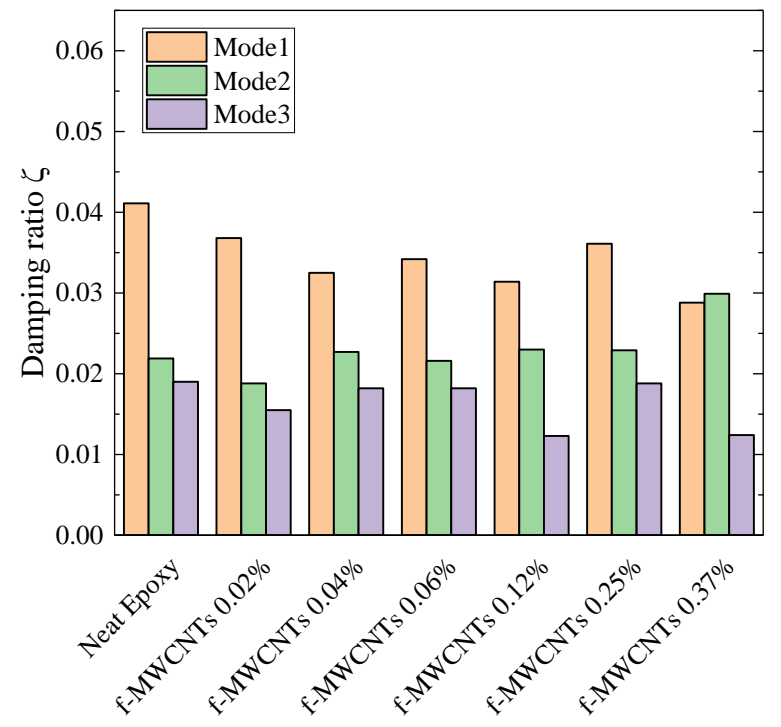

Fig. 7. Damping ratio of f-MWCNTs-reinforced composites with different loadings.

For the second mode of vibration, both pristine and functionalized multiwalled carbon nanotubes improved the damping characteristics of nanocomposite beams. There was 
over $40 \%$ improvement in damping ratio in p-MWCNTs/epoxy composites at $0.25 \mathrm{wt} . \%$ loading. The maximum improvement in damping ratio in f-MWCNTs/epoxy composites was observed at the 0.37 wt.\% loading and reached $36.5 \%$. Damping ratio results for the third mode of vibration showed no significant improvements.
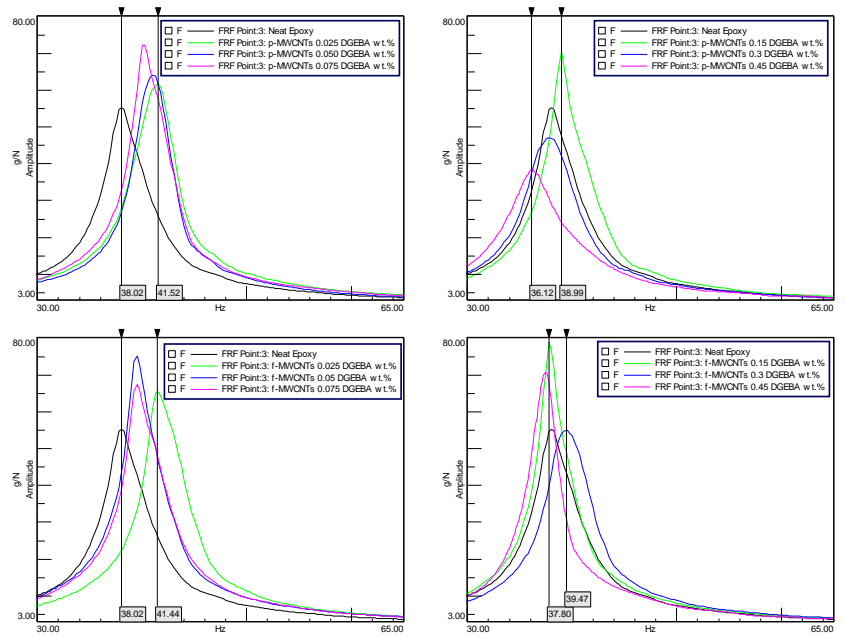

Fig. 8. Magnification of FRFs, coherence and phase diagrams around fundamental resonance peak of MWCNTs/epoxy.
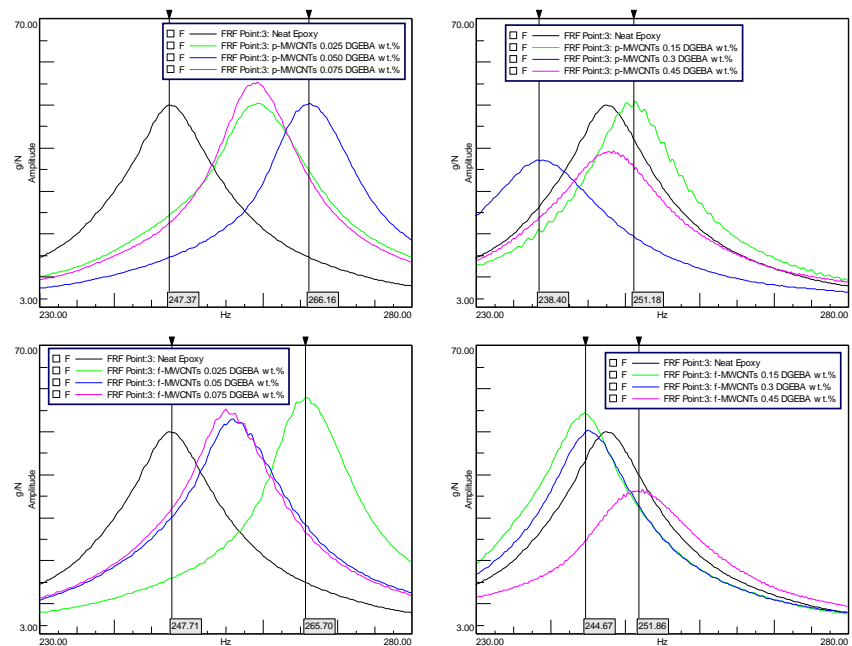

Fig. 9. Magnification of FRFs, coherence and phase diagrams around second resonance peak of MWCNTs/epoxy.

\section{CONCLUSION}

The experimental vibration and damping analyses of $\mathrm{p}$ MWCNTs/epoxy and f-MWCNTs/epoxy nanocomposites were completed. Nanocomposite specimens were fabricated for six different MWCNT loadings (0.02, 0.041, 0.061, 0.123, 0.25 and $0.37 \mathrm{wt} \%$ ). Microstructural analyses and tensile and bending tests were carried out to examine the effects of $p$ MWCNTs and f-MWCNTs. The frequency response functions, coherence and phase diagrams of nanocomposites were obtained using a periodic up-chirp forced vibration technique. The damped natural frequencies and damping ratios were obtained for different loadings of MWCNTs. The experimental results indicated that the damped natural frequencies of $\mathrm{p}$ MWCNTs/epoxy and f-MWCNTs/epoxy composites increased by adding CNTs up to 0.12 wt.\% and decreased with higher MWCNTs content. Another finding was that the improvement in fundamental natural frequency was more significant compared than that at higher natural frequencies. The forced vibration test also confirmed the beneficial effect of $p$ MWCNTs in improving the damping ratio of nanocomposites for the first and second vibration modes. However, a decrease in damping ratio was observed in f-MWCNTs/epoxy composites. For instance, a $46 \%$ improvement in damping ratio associated with fundamental vibration mode was observed at loadings of 0.37 wt.\% in p-MWCNTs/epoxy composites. While the damping ratios with f-MWCNTs at loadings of 0.020.06 wt.\% were still higher than with p-MWCNTs, they were not increasing at higher CNTs contents for the first mode of vibration.

\section{REFERENCES}

[1] M. Rafiee, X. Q. He, S. Mareishi, and K. M. Liew, "Modeling and stress analysis of smart CNTs/fiber/polymer multiscale composite plates," Int. J. Appl. Mech., vol. 6, no. 3, p. 1450025, 2014.

[2] M. Rafiee, F. Nitzsche, and M. Labrosse, "Dynamics, vibration and control of rotating composite beams and blades: A critical review," Thin-Walled Struct., vol. 119, pp. 795-819, 2017.

[3] I. Rafique, A. Kausar, and B. Muhammad, "Epoxy Resin Composite Reinforced with Carbon Fiber and Inorganic Filler: Overview on Preparation and Properties," Polym. Plast. Technol. Eng., vol. 55, no. 15, p. 03602559.2016.1163597, 2016.

[4] G. Mittal, V. Dhand, K. Y. Rhee, S.-J. Park, and W. R. Lee, “A review on carbon nanotubes and graphene as fillers in reinforced polymer nanocomposites," J. Ind. Eng. Chem., vol. 21, pp. 11-25, 2015.

[5] Fig. 11. Magnification of FRFs, coherence and phase diagrams around second resonance peak of MWCNTs/epoxy.

[6] H. Rajoria and N. Jalili, "Passive vibration damping enhancement using carbon nanotube-epoxy reinforced composites," Compos. Sci. Technol., vol. 65, no. 14, pp. 2079-2093, 2005.

[7] S. C. Her and S. W. Yeh, "Fabrication and Characterization of the Composites Reinforced with Multi-Walled Carbon Nanotubes," J. Nanosci. Nanotechnol., vol. 12, no. 10, pp. 8110-8115, 2012.

[8] K. Mehar, S. K. Panda, and T. R. Mahapatra, "Theoretical and experimental investigation of vibration characteristic of carbon nanotube reinforced polymer composite structure," Int. J. Mech. Sci., vol. 133, pp. 319-329, 2017. 